\title{
BLIND DECONVOLUTION WITH PSF REGULARIZATION FOR WIDE-FIELD MICROSCOPY
}

\author{
Margret Keuper ${ }^{\star \dagger} \quad$ Maja Temerinac-Ott ${ }^{\star \dagger} \quad$ Jan Padeken \\ Patrick Heun ${ }^{\ddagger}$ Thomas Brox ${ }^{\star \dagger} \quad$ Hans Burkhard $t^{\star \dagger} \quad$ Olaf Ronneberger ${ }^{\star \dagger}$ \\ ${ }^{\star}$ Computer Science Department, ${ }^{\dagger}$ BIOSS Centre of Biological Signalling Studies, \\ University of Freiburg, Germany \\ ${ }^{\ddagger}$ Max-Planck Institute for Immunobiology, Freiburg, Germany
}

\begin{abstract}
We propose to use a kernel intensity penalizer (KIP) in the blind maximum likelihood expectation maximization (MLEM) deconvolution scheme. With this very general kernel regularization term, we can stabilize the blind MLEM scheme even for the deconvolution of wide-field microscopic recordings. No complex prior point spread function models are needed. We combine state of the art optimization schemes using Tikhonov-Miller and TV regularization with our new kernel regularization. The proposed method improves the conventional deconvolution methods in terms of SNR on real and simulated datasets.
\end{abstract}

Index Terms - Wide-field microscopy, kernel penalizer, blind deconvolution, Total Variation, residual denoising.

\section{INTRODUCTION}

Wide-field microscopes are prevalently available and are, because of their low phototoxicity, capable to record living specimen over time. The disadvantage of wide-field recordings is the large amount of recorded defocused light that makes a deconvolution of the data indispensable for many applications. In order to reconstruct the specimen from the image, it is crucial to correctly estimate the point spread function (PSF) of the recording system. For non-blind deconvolution, PSF estimates can be generated by recording fluorescent beads or by theoretical models (e.g. [1]). However, the exact PSF depends on many parameters and can not be modeled completely.

Therefore, blind deconvolution techniques, that try to estimate both specimen and PSF from the recording, are appealing, even though the estimation of the large wide-field PSF is especially challenging. For the deconvolution of wide-field microscopic data, maximum likelihood expectation maximization (MLEM) deconvolution methods have proved to be very efficient $[2,3,4]$. However they are not convergent if no regularization is implemented [2]. Blind deconvolution methods even tend to converge to the trivial solution, where the convolution kernel (the estimated PSF) collapses to the $\delta$ impulse and the reconstructed specimen equals the recorded image. Thus, the regularization of the specimen function and the convolution kernel is necessary.

State of the art methods use prior models such as the Tikhonov Miller (TM) penalizer [5] or Total Variation (TV) regularization [6] on the specimen estimate, enforcing smoothness in the deconvolved data. [7] proposes to additionally use a residual denoising (RD) technique for specimen and PSF estimation as done before in [8] for the deconvolution of astronomical images. The regularization of the estimated PSF is more tricky, because no smoothness can be assumed.

Theoretical models have been employed for the kernel regularization. For example in [9] and [7] the kernel update is regularized by a projection of the current kernel estimate onto a theoretical PSF model [1]. Theoretical models however are cumbersome to compute and need to be adapted to every microscope and every specimen individually. In figure 1 , we show for example the influence of the refractive index of the specimen on the PSF.

In this paper, we propose to use the zero-order Tikhonov regularizer, that performs a kernel intensity penalization (KIP), in the blind MLEM deconvolution scheme. With this very general penalty term, we can easily avoid the trivial solution and stabilize the blind MLEM scheme. The introduction of a complex prior PSF model can be omitted. A similar kernel regularization has been used before in [10] for motion deblurring. However [10] use the $L_{2}$ norm for the data fitting term resulting in a different optimization scheme.

We have introduced the KIP stabilizer into the state of the art MLEM optimization schemes with TM and TV regularization on the data term as well as the optimization scheme with residual denoising. We show improvements of the deconvolution result for all three optimization schemes on simulated as well as on a real microscopic dataset.

This study was supported by the Excellence Initiative of the German Federal and State Governments (EXC 294) and the SFB(592). 
(a)



(b)

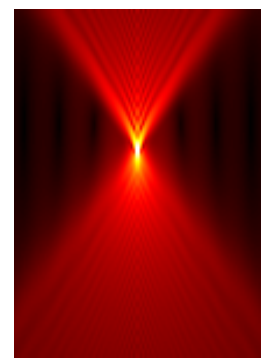

Fig. 1. YZ-section of simulated wide-field PSFs without (a) and with (b) spherical aberration with hot color map, generated with [11].

\section{BLIND DECONVOLUTION}

The image formation can be described by

$$
o=n(s * h)
$$

where the objective function $o: \Omega \subseteq \mathbb{R}^{3} \rightarrow \mathbb{R}$ denotes the recorded image, $s: \Omega \rightarrow \mathbb{R}$ is the specimen function, $h: \Omega \rightarrow \mathbb{R}$ the microscope specific point spread function (PSF), ' $*$ ' is the convolution operator and $n: \mathbb{R} \rightarrow \mathbb{R}$ is a voxelwise noise function. Generally, the PSF $h$ is assumed to integrate to one: $\int_{\Omega} h(\mathbf{x}) d \mathbf{x}=1$.

The aim of the blind MLEM deconvolution algorithm is to reconstruct $s$ and $h$ by finding $\hat{s}=\arg \max _{s}\{p(o \mid s, h)\}$ and $\hat{h}=\arg \max _{h}\{p(o \mid s, h)\}$. Since photon noise is Poisson distributed, $\hat{s}$ and $\hat{h}$ maximize the likelihood function [9]:

$$
p(o \mid s, h)=\prod_{\mathbf{x} \in \Omega} \frac{((s * h)(\mathbf{x}))^{o(\mathbf{x})} e^{-(s * h)(\mathbf{x})}}{o(\mathbf{x}) !}
$$

This likelihood can be maximized by minimizing functional

$$
J_{\text {MLEM }}(s, h)=\int_{\Omega}(s * h)(\mathbf{x})-o(\mathbf{x}) \cdot \log (s * h)(\mathbf{x}) d \mathbf{x},
$$

corresponding to the negative $\log$-likelihood $-\log (p(o \mid s, h))$. The resulting update scheme that minimizes eq. (3) is given by alternately updating [7]

$$
\hat{s}_{k+1}(\mathbf{x})=\hat{s}_{k}(\mathbf{x}) \cdot\left(\hat{h}_{k}^{m} * \frac{o}{\left(\hat{h}_{k} * \hat{s}_{k}\right)}\right)(\mathbf{x})
$$

and

$$
\hat{h}_{k+1}(\mathbf{x})=\frac{\hat{h}_{k}(\mathbf{x})}{\int_{\Omega} \hat{s}_{k}(\mathbf{x}) d \mathbf{x}} \cdot\left(\hat{s}_{k}^{m} * \frac{o}{\left(\hat{h}_{k} * \hat{s}_{k}\right)}\right)(\mathbf{x}) .
$$

where $s^{m}(\mathbf{x})=s(-\mathbf{x})$ is the mirrored specimen function and $h^{m}(\mathbf{x})=h(-\mathbf{x})$ is the mirrored PSF function.

Since the measured objective function $o$ is noisy, this update scheme can lead to amplified noise in the deconvolved data.
This effect can be reduced by denoising the residual $R_{k}$ [8] defined by

$$
o=\hat{s}_{k} * \hat{h}_{k}+R_{k} \text {. }
$$

$R_{k}$ can be denoised with any denoising function as for example wavelet denoising [8] or median filtering [7]. We follow [7] in using a $3 \times 3 \times 3$ median filter. With the denoised residual denoted by $\bar{R}_{k}=\operatorname{denoise}\left(R_{k}\right)$, the denoised objective function $\bar{o}_{k}=\hat{s}_{k} * \hat{h}_{k}+\bar{R}_{k}$ can be computed. $\bar{o}_{k}$ can be used instead of $o$ in the update scheme.

\section{DATA TERM REGULARIZATION}

\subsection{Tikhonov Miller Regularization}

To enforce smoothness in the reconstructed image, the quadratic Tikhonov-Miller regularizer can be employed. The energy functional becomes

$$
J_{\mathrm{TM}}(s, h)=J_{\mathrm{MLEM}}(s, h)+\lambda_{\mathrm{TM}} \int_{\Omega}|\nabla s(\mathbf{x})|^{2} d \mathbf{x}
$$

where $\lambda_{\mathrm{TM}}$ is the weighting for the regularization. The TM regularized specimen estimation update scheme is then:

$$
\hat{s}_{k+1}(\mathbf{x})=\frac{\hat{s}_{k}(\mathbf{x})}{1-2 \lambda_{\mathrm{TM}} \Delta \hat{s}_{k}(\mathbf{x})} \cdot\left(\hat{h}_{k}^{m} * \frac{o}{\left(\hat{h}_{k} * \hat{s}_{k}\right)}\right)(\mathbf{x}) .
$$

where $\Delta s=\frac{\partial^{2} s}{\partial x_{1}^{2}}+\frac{\partial^{2} s}{\partial x_{2}^{2}}+\frac{\partial^{2} s}{\partial x_{3}^{2}}$.

\subsection{Total Variation Regularization}

An alternative is total variation (TV) regularizer. TV does not enforce smoothness but piecewise constancy. Thus, it depends on the specimen which of the two models performs best. The energy functional with TV regularizer on the data term is

$$
J_{\mathrm{TV}}(s, h)=J_{\mathrm{MLEM}}(s, h)+\lambda_{\mathrm{TV}} \int_{\Omega}|\nabla s(\mathbf{x})| d \mathbf{x}
$$

where $\lambda_{\mathrm{TV}}$ is the weighting for TV regularization. The TV regularized specimen estimation update scheme is given by:

$$
\hat{s}_{k+1}(\mathbf{x})=\frac{\hat{s}_{k}(\mathbf{x})}{1-\lambda_{\mathrm{TV}} \operatorname{div}\left(\frac{\nabla s(\mathbf{x})}{|\nabla s(\mathbf{x})|}\right)} \cdot\left(\hat{h}_{k}^{m} * \frac{o}{\left(\hat{h}_{k} * \hat{s}_{k}\right)}\right)(\mathbf{x}),
$$

where $\operatorname{div}(s)=\frac{\partial s}{\partial x_{1}}+\frac{\partial s}{\partial x_{2}}+\frac{\partial s}{\partial x_{3}}$.

\section{KERNEL INTENSITY PENALIZER}

To stabilize the deconvolution kernel, we add a simple Tikhonov stabilizer of zero order to the energy functional. With this regularization, we can avoid the trivial solution 
$\mathrm{h}=\delta$-impulse. The kernel intensity penalized energy functional becomes

$$
J_{\mathrm{KIP}}(s, h)=J_{\mathrm{MLEM}}(s, h)+\lambda_{\mathrm{KIP}} \int_{\Omega} h(\mathbf{x})^{2} d \mathbf{x},
$$

where $\lambda_{\text {KIP }}$ is the weighting for the intensity penalty regularization of the kernel. The resulting update scheme for the kernel is given by

$$
\hat{h}_{k+1}(\mathbf{x})=\frac{\hat{h}_{k}(\mathbf{x}) \cdot\left(\hat{s}_{k}^{m} * \frac{o}{\left(\hat{h}_{k} * \hat{s}_{k}\right)}\right)(\mathbf{x})}{\int_{\Omega} \hat{s}_{k}(\mathbf{y}) d \mathbf{y}+2 \cdot \lambda_{\mathrm{KIP}} h(\mathbf{x})} .
$$

\section{EVALUATION}

We have evaluated our new kernel penalizer in three different blind deconvolution settings:

- Residual denoising in both specimen and kernel update (RD RD),

- TM regularization for the specimen and residual denoising for the kernel update (TM RD),

- TV regularization for the specimen and residual denoising for the kernel update (TV RD).

All three settings have been evaluated on simulated and on real microscopic data.

\subsection{Simulated Data}

For authentic specimen simulations, we used the HeLa Cell Nucleus simulation tool from [12]. The central slice from the simulated cell nucleus is shown in fig. 3(a). For the recording, we assumed a background fluorescence of $1.2 \%$ of the maximum specimen fluorescence. The specimen was blurred with an ideal wide-field PSF without aberration downloaded from ${ }^{1}$. The PSF is generated assuming a refractive index of 1.518 , numerical aperture NA $=1.4$, wavelength $\lambda=530 \mathrm{~nm}$, spatial resolution $\delta r=64.5 \mathrm{~nm}$ and an axial resolution $\delta z=160 \mathrm{~nm}$.

The simulated HeLa Cell nucleus was first blurred with this PSF. Then, we added two different levels of Poisson noise, resulting in a SNR of 27.552 (SIM 1) and 18.588 (SIM 2)(between the blurred image before adding noise and the final recording simulations). The SNR between the ground truth and the final simulations is 1.628 (SIM 1) and 1.6135 (SIM 2) respectively.

We also simulated the PSF estimation from the bead recording. The bead recording was simulated by convolving a small sphere with a radius of $90 \mathrm{~nm}$ with the PSF. A background fluorescence of $1 \%$ of the bead fluorescence $L$ was assumed. Then, we added Poisson noise. The resulting SNR was 2.9856 .

\footnotetext{
${ }^{1}$ http: / / bigwww.epfl. ch/deconvolution/ ?p=bead
}

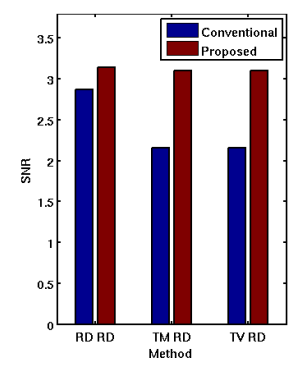

(a) SIM 1

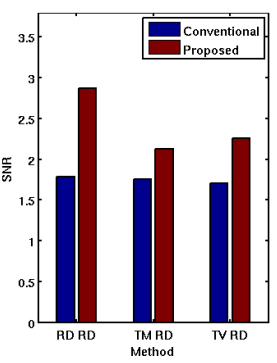

(b) SIM 2

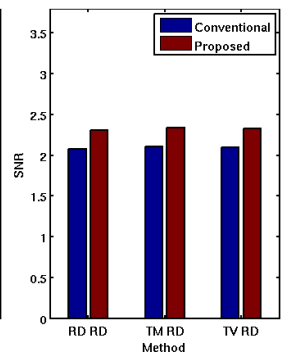

(c) MIC
Fig. 2. Resulting SNR with and without KIP.

\subsection{Real Data}

The actual recordings where taken from a fixed DAPI stained Drosophila S2 cell nucleus. For the wide-field microscope, we used a resolution of $0.0642 \times 0.0642 \mu \mathrm{m}$ in $\mathrm{x}-\mathrm{y}$ direction and $0.2 \mu \mathrm{m}$ in z-direction. With the same microscope settings and resolution, we also recorded a TetraSpeck $0.2 \mu \mathrm{m}$ bead in order to generate an initial PSF estimate.

The identical Drosophila S2 cell nucleus was recorded with a spinning disk confocal microscope for comparison. Since the PSF of the spinning disk microscope is very small, this data serves us as ground truth for the wide-field deconvolution. The spinning disk recording was taken with a resolution of $0.1 \times 0.1 \mu \mathrm{m}$ in $\mathrm{x}$-y direction and $0.2 \mu \mathrm{m}$ in z-direction. The two recordings were registered using affine registration from 2 . The central slice of the spinning disk recording after registration is shown in fig. 4(a), the same slice of the wide-field recording is displayed in fig. 4(b). The SNR computed between the recordings from the different modalities is 0.9229 .

\subsection{Results}

We have tested weightings $\lambda_{\mathrm{TM}}$ and $\lambda_{\mathrm{TV}}$ between $10^{-7}$ and $10^{-3}$ for the two simulated datasets (SIM1 and SIM2) and for the real data (MIC). The best results for SIM 1 were achieved with $\lambda_{\mathrm{TM}}=10^{-7}$ and $\lambda_{\mathrm{TV}}=5 \cdot 10^{-3}$. For SIM $2 \lambda_{\mathrm{TM}}=$ $5 \cdot 10^{-6}$ and $\lambda_{\mathrm{TV}}=10^{-3}$ worked best. For the real data, we found that $\lambda_{\mathrm{TM}}=10^{-7}$ and $\lambda_{\mathrm{TV}}=10^{-3}$ performed best. For the kernel penalizer, we have chosen the relative weighting $\lambda_{\mathrm{KIP}}=6 \cdot \int_{\Omega} o(\mathbf{x}) d \mathbf{x}$ for all three datasets. After 400 iterations, we have found that the energy is converged for all methods. The resulting SNR for the three different methods with and without kernel intensity regularizer is plotted in fig. 2. All methods were improved by the new regularization term. The results on simulated data can be seen in fig. 3 (d)(e), and (f), the results on real data are displayed in fig. 4(c)(d), and (e). Without the kernel penalizer, spurious edges from outof-focus planes are visible, that can be eliminated with our new approach. Additionally, the contrast within the nucleus is much better with our method.

\footnotetext{
${ }^{2}$ http://www.fil.ion.ucl.ac.uk/spm/software/
} 


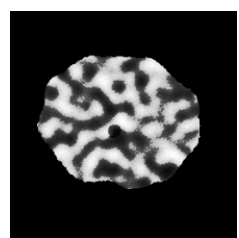

(a) Simulated cell (Ground Truth)

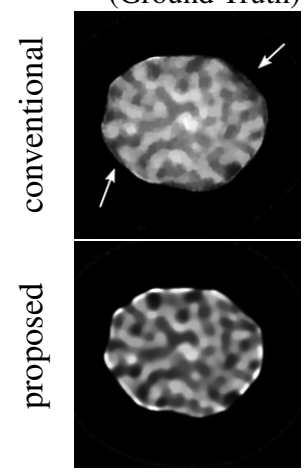

(d) TV RD

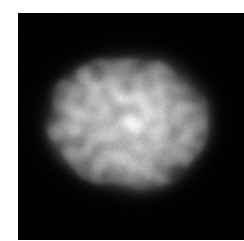

(b) SIM 1

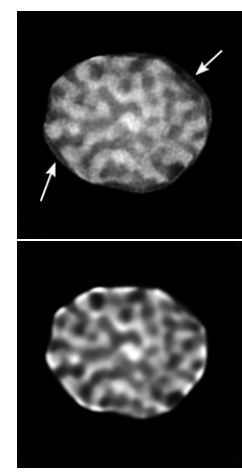

(e) TM RD

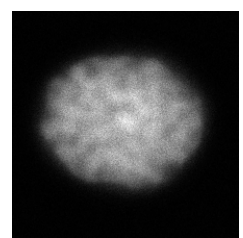

(c) SIM 2

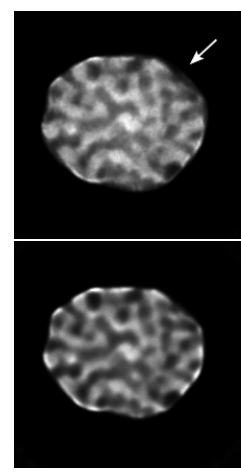

(f) RD RD

Fig. 3. Simulated data and results on SIM 1. The arrows indicate artificially introduced edges from out-of-focus planes.

\section{CONCLUSION}

We have introduced a new kernel penalizer into the blind MLEM deconvolution framework. This penalizer avoids the trivial solution in the blind deconvolution framework and has improved the deconvolution results on simulated as well as on real data. The penalizer has also shown to be beneficial in combination with TM and TV regularization as well as with a residual denoising technique.

\section{REFERENCES}

[1] F. S. Gibson and F. Lanni, "Experimental test of an analytical model of aberration in an oil-immersion objective lens used in three-dimensional light microscopy," $J$. Opt. Soc. Am. A, vol. 8, pp. 1601-1613, 1991.

[2] P. Sarder and A. Nehorai, "Deconvolution methods for 3-d fluorescence microscopy images," Signal Processing Magazine, IEEE, vol. 23, no. 3, pp. 32-45, May 2006.

[3] J.-A. Conchello and J. G. Mcnally, "Fast regularization technique for expectation maximization algorithm for optical sectioning microscopy," in Proc. SPIE 2655, 1996, pp. 199-208.

[4] T. J. Holmes, "Blind deconvolution of quantum-limited incoherent imagery: maximumlikelihood," J. Opt. Soc. Am. A, vol. 9, pp. 10521061, July 1992.

[5] G. M. P. vanKempen, P. J. van Vliet, L. J.and Verveer, and H. T. M vanderVoort, "A quantitative comparison

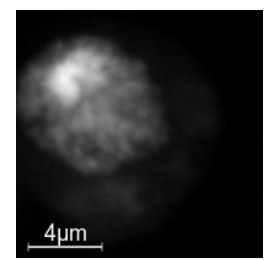

(a) Spinning Disk (Ground Truth)

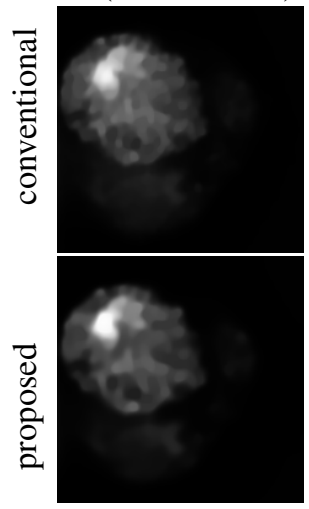

(c) TV RD

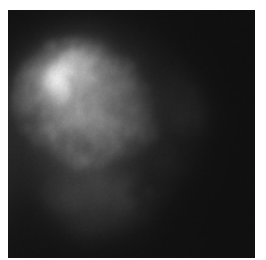

(b)Wide-field

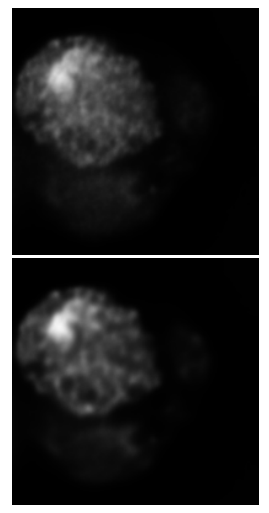

(d) TM RD

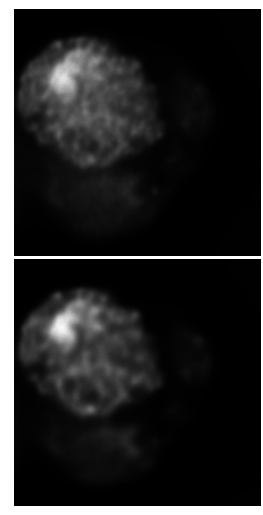

(e) RD RD
Fig. 4. Results on a Drosophila S2 cell nucleus recording.

of image restoration methods for confocal microscopy," J. of Mic., vol. 185, pp. 354-365, 1997.

[6] N. Dey, L. Blanc-Feraud, C. Zimmer, P. Roux, Z. Kam, J.C. Olivo-Marin, and J. Zerubia, "Richardsonlucy algorithm with total variation regularization for $3 \mathrm{~d}$ confocal microscope deconvolution," Micr. Research and Technique, vol. 69, pp. 260-66, 2006.

[7] T. Kenig, Z. Kam, and A. Feuer, "Blind image deconvolution using machine learning for three-dimensional microscopy," IEEE Trans. on PAMI, vol. 32, no. 12, pp. 2191-2204, 2010.

[8] J.-L. Starck, E. Pantin, and F. Murtagh, "Deconvolution in astronomy: a review," Pub. of the Astron. Soc. of the Pac., vol. 114, pp. 1051-1069, 2002.

[9] J. Markham and J.-A. Conchello, "Parametric blind deconvolution: a robust method for the simultaneous estimation of image and blur," J. Opt. Soc. Am. A, vol. 16, no. 10, pp. 2377-2391, Oct 1999.

[10] S. Cho and S. Lee, "Fast motion deblurring," $A C M$ Trans. Graph., vol. 28, pp. 145:1-145:8, 2009.

[11] H. Kirshner, D. Sage, and M. Unser, "3d psf models for fluorescence microscopy in imagej," in MAF'12, 2011.

[12] D. Svoboda, M. Kozubek, and S. Stejskal, "Generation of digital phantoms of cell nuclei and simulation of image formation in 3d image cytometry," Cytometry Part $A$, vol. 75A, no. 6, 2009. 